\title{
Aspects cliniques et pratiques de la sédation par inhalation de MEOPA en implantologie chez les patients adultes anxieux, phobiques ou à risques médicaux
}

\author{
Frédéric Philippart (Paris)
}

fredericphilippart@dental-implant.fr

La sédation au MEOPA permet de répondre efficacement à l'anxiété des patients adultes et certainement de les inciter à venir consulter plus tôt, mais elle permet aussi de diminuer les risques de malaises vagaux lors des interventions.

La pratique montre que la pression artérielle et le rythme cardiaque baissent significativement, lorsque les soins sont réalisés sous sédation par MEOPA.

Il est probable que la conjugaison de l'effet relaxant du MEOPA et de la supplémentation en oxygène apportée par ce mélange, ait un effet bénéfique chez les patients à risque cardio- vasculaire (ischémie myocardique, hypertension artérielle).

La mise en place de cette technique de sédation dans le cadre d'un exercice habituel de cabinet, orienté ou non vers la chirurgie buccale, nécessite de maitriser des protocoles de prise en charge des patients adultes mais aussi de gérer l'ergonomie du système d'administration du MEOPA dans un environnement caractéristique de soins dentaires ou de chirurgie buccale.

Nous verrons comment un chirurgien-dentiste peut intégrer cette technique de sédation dans son exercice habituel, nous décrirons le matériel spécifique dont il dispose ainsi que le protocole d'administration, et nous envisagerons la manière dont le contrôle de l'anxiété au cabinet dentaire s'inscrit dans le cadre d'une prise en charge globale du patient adulte. 